\title{
Association between physical activity and urinary incontinence during pregnancy and postpartum: a systematic review and meta-analysis
}

\author{
Nadine von Aarburg ${ }^{1}$, Nikolaus Veit-Rubin², Michel Boulvain ${ }^{3}$, Jeanne Bertuit ${ }^{4}$, Colin \\ Simonson $^{5}$, and David Desseauve ${ }^{6}$ \\ ${ }^{1} \mathrm{HES}-\mathrm{SO}$ \\ ${ }^{2}$ Medical University of Vienna \\ ${ }^{3}$ University of Geneva \\ ${ }^{4} \mathrm{HESAV}$ \\ ${ }^{5}$ Hopital du Valais \\ ${ }^{6}$ Centre Hospitalier Universitaire Vaudois
}

October 28, 2020

\begin{abstract}
Background The impact of physical activity during pregnancy and postpartum on urinary incontinence in women is unclear. Objectives To assess the association of physical activity and urinary incontinence during pregnancy and postpartum in a systematic review of the literature. Search Strategy A search of publications indexed in five major electronic databases (CENTRAL, PubMed, EMBASE, CINAHL and PEDro) was performed from their respective inception dates to the 30 March 2020 with a combination of keywords to identify studies of interest. Google Scholar was used for non-indexed literature. Selection Criteria All studies comparing physical activity with standard care in pregnant and postpartum women were selected. Data collection and Analysis Two reviewers, independently, selected studies, assessed quality and extracted data. Odds ratio with 95\% confidence intervals were calculated using fixed effects or random effects meta-analyses (Mantel-Haenszel method), for low and moderate heterogeneity between studies, respectively. Main Results Seven studies $(\mathrm{n}=12479)$ were included. Data of four studies could be pooled for meta-analyses; subgroup and sensitivity analyses were not possible. Physical activity, either during pregnancy or postpartum, is not associated with urinary incontinence, OR 0.90 (95\% CI: 0.69-1.18) and OR 1.31 (95\% CI: $0.74-2.34$ ), respectively. Conclusions The 'low' quality of evidence (GRADE) does not show that physical activity during pregnancy or postpartum has any impact on urinary incontinence. Moderate physical activities should therefore be encouraged for the evidence-based benefits associated on other obstetrical outcomes. Funding None to declare. Keywords Pregnancy; postpartum; urinary incontinence; physical activity; exercise
\end{abstract}

\section{Hosted file}

Main Document.pdf available at https://authorea.com/users/370880/articles/489400-associationbetween-physical-activity-and-urinary-incontinence-during-pregnancy-and-postpartum-asystematic-review-and-meta-analysis 

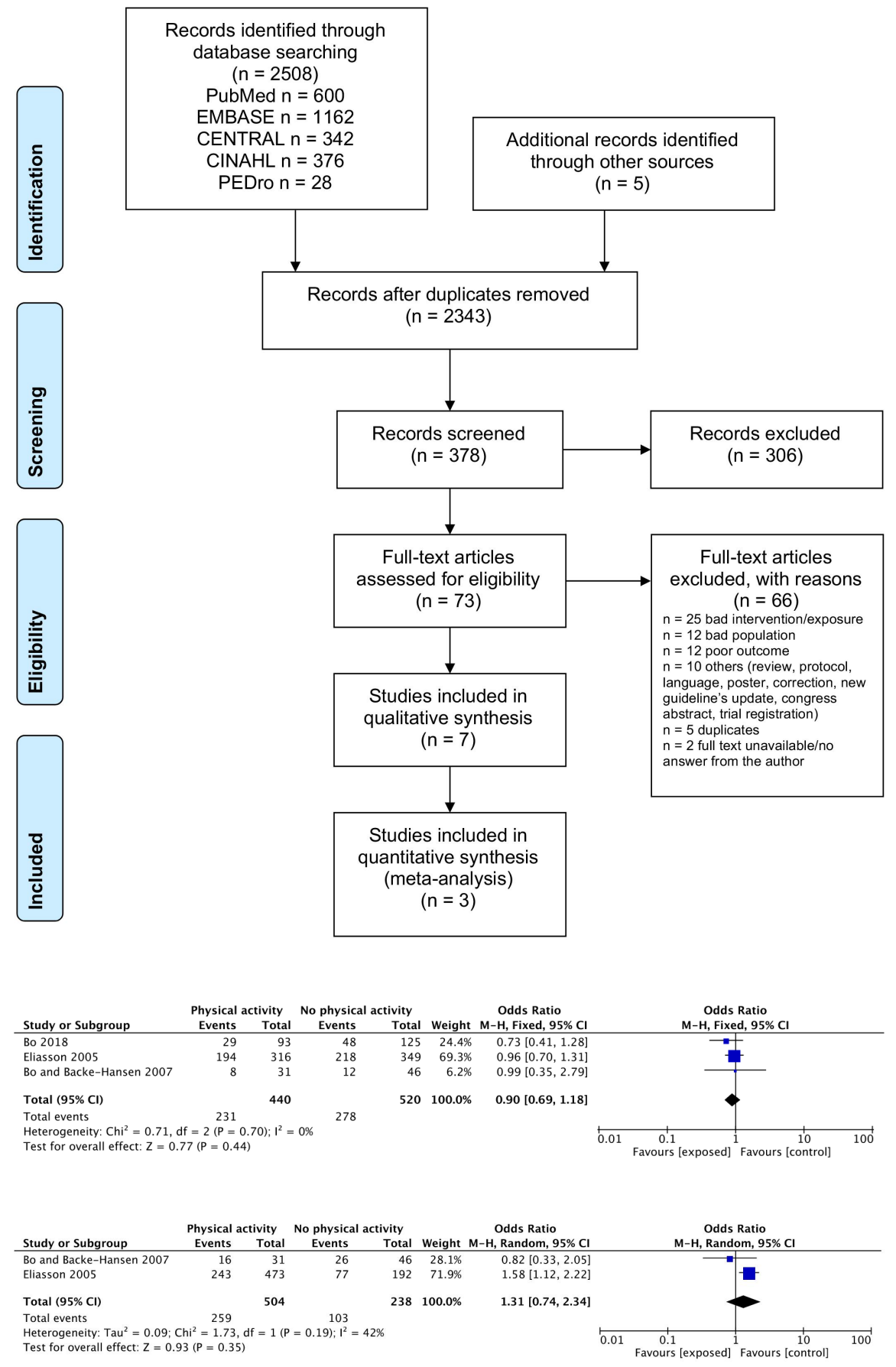


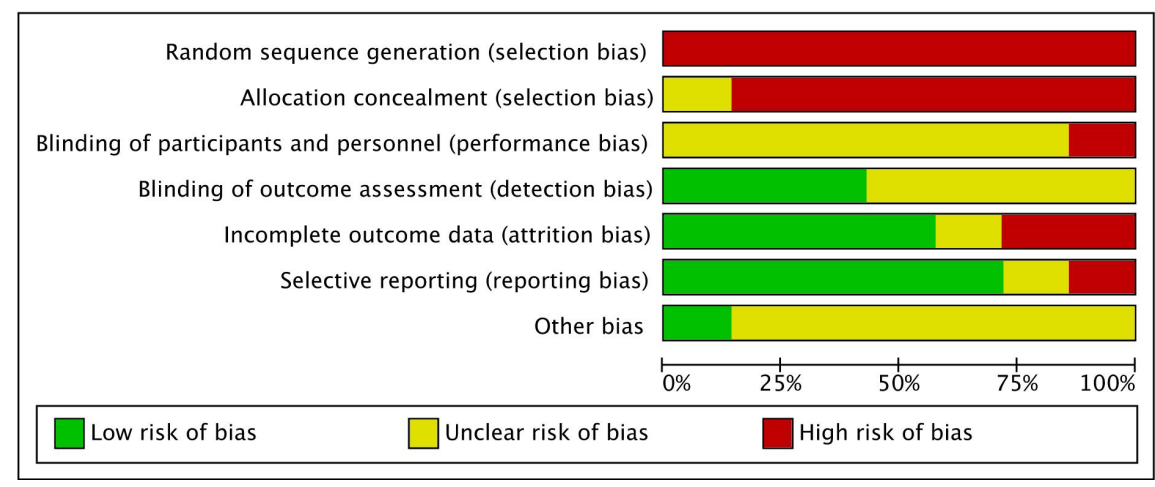

